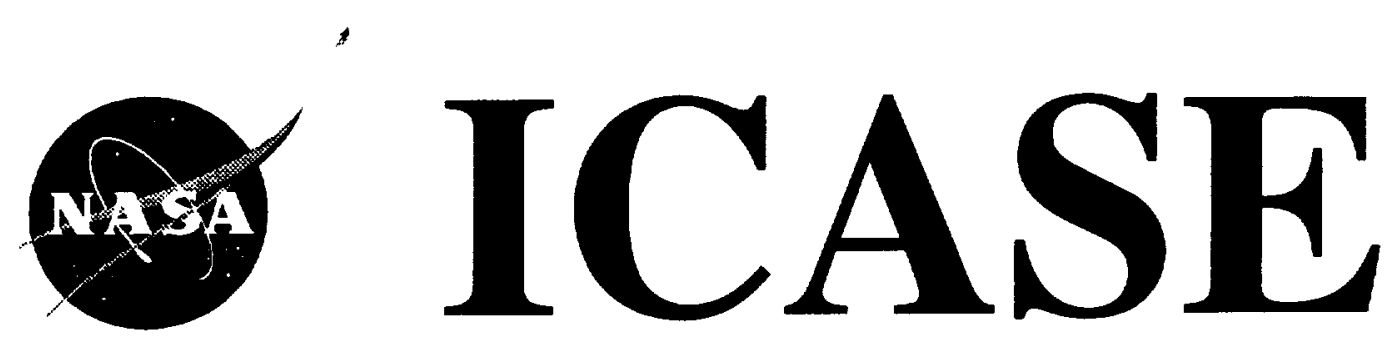

\title{
REPRESENTATION OF FEEDBACK OPERATORS FOR HYPERBOLIC SYSTEMS
}

John A. Burns

Belinda B. King

(NASA-CR-198171) REPRESENTATION OF
FEEDBACK OPERATORS FOR HYPERBOLIC
SYSTEMS FInAl RePOFt (ICASE) $14 \mathrm{p}$

N95-31319

Unclas

G3/64 0058497

Contract No. NAS1-19480

May 1995

Institute for Computer Applications in Science and Engineering NASA Langley Research Center

Hampton, VA 23681-0001

SRA Operated by Universities Space Research Association 


\title{
REPRESENTATION OF FEEDBACK OPERATORS FOR HYPERBOLIC SYSTEMS
}

\author{
John A. Burns* \\ Center for Optimal Design and Control \\ Interdisciplinary Center for Applied Mathematics \\ Virginia Tech \\ Blacksburg, Virginia 24061 \\ Belinda B. King ${ }^{\dagger}$ \\ Department of Mathematics \\ Oregon State University \\ Corvallis, Oregon 97331
}

\begin{abstract}
We consider the problem of obtaining integral representation of feedback operators for damped hyperbolic control systems. We show that for the wave equation with Kelvin-Voigt damping and non-compact input operator, the feedback gain operator is Hilbert-Schmidt. This result is then used to provide an explicit integral representation for the feedback operator in terms of functional gains. Numerical results are given to illustrate the role that damping plays in the smoothness of these gains.
\end{abstract}

* This research was supported in part by the Air Force Office of Scientific Research under grant F49620-93-1-0280 and by the National Aeronautics and Space Administration under contract No. NASA-19480 while the author was a visiting scientist at the Institute for Computer Applications in Science and Engineering, NASA Langley Research Center, Hampton, VA $23681-0001$.

This research was supported in part by the Air Force Office of Scientific Research under grant F49620-93-1-0280 while the author was a visiting scientist at the Air Force Center for Optimal Design and Control, Virginia Polytechnic Institute and State University, Blacksburg, VA 24061-0531, and by the National Science Foundation under grant DMS-9409506. 



\section{Introduction and Motivation}

The purpose of this article is to extend the representation theorem in [1] and [7] to certain classes of damped hyperbolic systems. The original motivation for our study of hyperbolic systems comes from the work by Lupi, Chun, and Turner [8]. The approach in [8] is interesting because they make no prior assumptions regarding the form of the controls and actuators so that the gains operators produced by an optimal design could be used to make decisions about where actuators and sensors are best placed. In particular, in [8] it was assumed that the input operator was the identity and the elastic system was not damped. By solving the LQR problem with the input operator equal to the identity, one can gain insight into the type and location of practical distributed controllers for structural control. This insight comes from explicit knowledge of the kernels (so called functional gains) that describe the integral representations of feedback gain operators. Even with no damping the LQR problem has a solution since the input operator is the identity (the system is exactly controllable). However, as we see below the problem with no damping is extremely complex. Basic questions concerning the existence and smoothness of functional gains remain open and yet these issues are important in the applications proposed in [8]. Consequently, as a first step we take the middle ground and consider damped systems with distributed control.

Except for the obvious cases with bounded input operator and a finite number of controllers, the problem of obtaining explicit representations of feedback laws is more complex than one might first imagine. In the most general case, this problem is equivalent to the problem that led Grothendieck to develop the theory of topological tensor products and nuclear spaces. This theory led to the famous Schwartz Kernel Theorem. However, the fact that the operators of interest often arise as solutions to Riccati equations can be exploited to yield reasonable results. The representation problem for a parabolic problem with unbounded control operator was first considered in [1] and [7]. It was shown that as long as the input operator is bounded relative to the open loop dynamic operator, the solution to the algebraic Riccati equation is Hilbert-Schmidt. This fact was then exploited to show that the resulting feedback operator had an integral representation. For the hyperbolic case considered here, these types of results are more intricate and highly dependent on the type of damping.

In the Section 2 we present a numerical example involving the control of a hybrid cable-mass system. This example is used to motivate the model problem and to demonstrate that distributed controllers can enhance disturbance attenuation. We then concentrate on the 1D wave equation with Kelvin-Voigt damping. Although similar results can be obtained for other damping models, we present the basic theorem for this model in order to keep this paper moderately short. However, we illustrate the ideas and difficulties for other damping models with numerical examples. Finally we close with a few comments about future work and other open problems.

\section{Control for a Cable-Mass System}

The following system was proposed by Nayfeh, Nayfeh and Mook [11] as a simple example of a nonlinear distributed parameter system with the property that many standard discretized lumped models failed to capture the essential nonlinear behavior of the dynamic system governed by the partial differential equation. This system was also considered in [2] where it was used as a test model for MINMAX control for disturbance attenuation. However, in [2] there was only one controller and, although disturbance attenuation was achieved, we shall see below adding a distributed controller can improve performance. The MINMAX approach provides a "robust state feedback control law" which is less sensitive to disturbances and certain unmodeled dynamics than is the LQR design. The idea is to obtain a representation of the control law and then use approximation theory to compute finite dimensional suboptimal controllers. These suboptimal controllers were used to attack the problem of designing reduced order state estimators. We shall limit our discussion here to the full state feedback problem.

Consider the hybrid nonlinear distributed parameter system described by a vibrating cable held fixed at one end and with a mass attached at the other end. The mass is suspended by a spring which has nonlinear stiffening terms and is forced by a disturbance (see Figure 2.1). The equations for the hybrid system are [2] 


$$
\begin{aligned}
& \rho \frac{\partial^{2}}{\partial t^{2}} w(t, s)=\frac{\partial}{\partial s} {\left[\tau \frac{\partial}{\partial s} w(t, s)+\gamma \frac{\partial^{2}}{\partial t \partial s} w(t, s)\right]+\rho u_{1}(t, s) } \\
& 0<s<\ell, t>0 \\
& m \frac{\partial^{2}}{\partial t^{2}} w(t, \ell)=- {\left[\tau \frac{\partial}{\partial s} w(t, \ell)+\gamma \frac{\partial^{2}}{\partial t \partial s} w(t, \ell)\right] } \\
&-\alpha_{1} w(t, \ell)-\alpha_{3}[w(t, \ell)]^{3}+\eta(t)+m u_{2}(t)
\end{aligned}
$$

with boundary condition

$$
w(t, 0)=0
$$

The initial conditions are given by

$$
w(0, s)=w_{0}, \quad \frac{\partial}{\partial t} w(0, s)=w_{1} .
$$

Here, $w(t, s)$ represents the displacement of the cable at time $t$, and position $s, w(t, \ell)$ represents the position of the mass at time $t, \rho$ and $m$ are the densities of the cable and mass respectively, $\tau$ is the tension in the cable, and $\gamma$ is a damping coefficient. The alphas are coefficients describing the nonlinear effects of the spring. The term $\eta(t)$ is viewed as a disturbance and $u_{1}(t, s)$ and $u_{2}(t)$ are control inputs. For the moment we assume Kelvin-Voigt (internal) damping in the cable.

The problem is hybrid in that the system is described by a linear partial differential equation (the wave equation) coupled through the boundary condition to a low order nonlinear ordinary differential equation (Duffing's equation). In [2] it was assumed that the control acted exclusively on the mass (i.e. that $u_{1}(t, x)$ $=0)$.

This model is often first written as a second order system in a Hilbert space $H$ of the form

$$
\ddot{y}(t)+D_{0} \dot{y}(t)+A_{0} y(t)+F_{0}(y(t))=B_{0} u(t) .
$$

For the cable-mass problem considered here, $H=L_{2}(0,1) \times \mathbb{R}^{1}$ and $y(t)=[w(t, \cdot), w(t, \ell)]^{T}$.

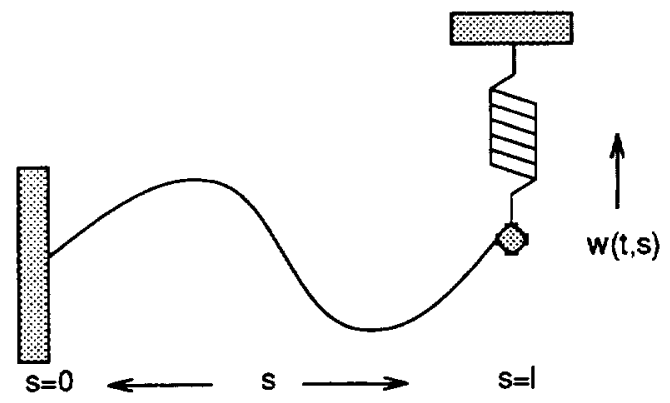

Figure 2.1: Cable-Mass System

This formal system has the advantage that it has the same appearance as the finite dimensional case and in order to address viscous and "structural" damping one merely replaces $D_{0}=A_{0}$ with $D_{0}=I$ and $D_{0}=\left[A_{0}\right]^{1 / 2}$, respectively. We note however, that it is more consistent with physics to write the system in the form

$$
\ddot{y}(t)+S^{*}(S y(t)+\gamma T \dot{y}(t))+F_{0}(y(t))=B_{0} u(t)
$$

where $S=T=\left[A_{0}\right]^{1 / 2}$. Observe that $A_{0}=A_{0}^{*}>0$ and so $S^{*}=S$ and $S^{*} S=S^{*} T=A_{0}$. Hence, (2.6) is formally obtained by factoring $\left[A_{0}\right]^{1 / 2}$ out of the expression $D_{0} \dot{y}(t)+A_{0} y(t)$ in (2.5). Note also that (2.6) is of a form that allows for structural damping where $S=\left[A_{0}\right]^{1 / 2}$ and $T=I$, as well as for viscous damping where $S=\left[A_{0}\right]^{1 / 2}$ and $T=S^{-1}=\left[A_{0}\right]^{-1 / 2}$. In addition, by writing the system in the second order form (2.6), one captures a form that comes from balance laws and at the same time sets the stage for a simple formulation of the problem in first order state space form. 
The system governed by equations (2.1) - (2.4) can be written as a dynamical system in an appropriate (infinite dimensional) state space. Although there are several equivalent formulations for this problem, we shall write the governing equations as the first order system

$$
\dot{x}(t)=A x(t)+F(x(t))+B u(t)+D \eta(t), \quad x(0)=x_{0}
$$

where at time $t$ the state $x(t)=[y(t), \dot{y}(t)]^{T}$ lies in the Hilbert space $X=H_{L}^{1} \times \mathbb{R} \times L_{2} \times \mathbb{R}$. Here, $H_{L}^{1}$ is the subspace of the Sobolev space $H^{1}=H^{1}[0, \ell]$ defined by $H_{L}^{1}=\left\{w \in H^{1}: w(0)=0\right\}$, and $L_{2}$ is the standard Lebesgue space of square integrable functions. The control $u(t)$ lies in the control space $U=L_{2} \times \mathbb{R}$. Here the inner product in $X$ is

$$
\begin{gathered}
\left\langle[w(\cdot), \xi, v(\cdot), \mu]^{T},[\hat{w}(\cdot), \hat{\xi}, \hat{v}(\cdot), \hat{\mu}]^{T}\right\rangle=\tau \int_{0}^{\ell} w^{\prime}(x) \hat{w}^{\prime}(x) d x+\alpha_{1} \xi \hat{\xi} \\
+\rho \int_{0}^{\ell} v^{\prime}(x) \hat{v}^{\prime}(x) d x+m \mu \hat{\mu}
\end{gathered}
$$

It is important to precisely define the system operators and their domains in order to obtain correct representations of the feedback operators that will be used to control the system. Let $\delta_{\ell}$ denote the "evaluation operator" defined on $H^{1}[0, \ell]$ by $\delta_{\ell}(\phi(\cdot))=\phi(\ell)$ and define the linear operator $A$ on the domain $\mathcal{D}(A) \subseteq X$ by

$$
\begin{gathered}
\mathcal{D}(A)=\left\{x=[w, \xi, v, \mu]^{T} \in X: w, v \in H_{L}^{1},\left\{\frac{\tau}{\rho} \frac{d}{d s} w+\frac{\gamma}{\rho} \frac{d}{d s} v\right\} \in H^{1},\right. \\
w(\ell)=\xi, v(\ell)=\mu\},
\end{gathered}
$$

and

$$
A x=\left[v, \mu, \frac{d}{d s}\left\{\frac{\tau}{\rho} \frac{d}{d s} w+\frac{\gamma}{\rho} \frac{d}{d s} v\right\},-\delta_{\ell}\left\{\frac{\tau}{m} \frac{d}{d s} w+\frac{\gamma}{m} \frac{d}{d s} v\right\}-\frac{\alpha_{1}}{m} \xi\right]^{T} .
$$

The control input operator $B$ and the disturbance operator $D$ are defined by

$$
B=\left[0, I_{H}\right]^{T} \text { and } D \eta=\left[0,0,0, \frac{1}{m} \eta\right]^{T},
$$

respectively. The nonlinear operator $F$ is defined on $X$ by

$$
F(x)=\left[0,0,0,-\frac{\alpha_{3}}{m}[\xi]^{3}\right]^{T}=\left[0, F_{0}(y)\right]^{T} .
$$

Observe that the input operator is the same as that used in [8] in their analysis.

As noted above, this problem with $u_{1}(t, s)=0$ was considered in [2] where MINMAX control was used to design a low order dynamics control law. This law was based on two outputs (position and velocity of the mass) and resulted in a practical low order design. For this note, we shall consider only the full state feedback problem. However, we allow for distributed control through $u_{1}(t, s)$. The simplest approach is to linearize the system, use MINMAX design to obtain a feedback operator and apply this law to the full plant.

The linearized system has the form

$$
\frac{d}{d t} x(t)=A x(t)+B u(t)+D \eta(t), \quad x(0)=x_{0} .
$$

For this problem, with Kelvin-Voigt damping, one can apply the MINMAX theory in [10] to obtain a feedback law of the form

$$
u(t)=\left[\begin{array}{c}
u_{1}(t, s) \\
u_{2}(t)
\end{array}\right]=-K_{\theta} x(t)
$$

where for $\theta \geq 0$ the gain operator $K_{\theta}: X \rightarrow H$ is given by

$$
K_{\theta}=B^{*} P_{\theta}
$$


and $P_{\theta}$ satisfies the algebraic Riccati equation

$$
A^{*} P+P A-P\left[B B^{*}-\theta^{2} M\right] P+Q=0 .
$$

Here $M=M^{*} \geq 0, Q=Q^{*} \geq 0$ and the system (2.16) holds in the weak sense (see [10]). When $\theta=0$ one has the LQR design. However, when $\theta>0$ the corresponding MINMAX controller provides additional disturbance attenuation (see [2]).

Figure 2.2 clearly illustrates the difference between the performance achieved in [2] and what one can achieve with additional distributed control. For this example, we use the same parameters and finite element scheme found in [2].

\begin{tabular}{||c|c|c|c|c|c|c|c||}
\hline \hline$\rho$ & $\tau$ & $\gamma$ & $m$ & $\ell$ & $\alpha_{1}$ & $\alpha_{2}$ & $\alpha_{3}$ \\
\hline 1 & 1 & .005 & 3 & 2 & .01 & 0 & 3 \\
\hline \hline
\end{tabular}

When there is control on the mass only, the maximum value of $\theta$ yielding a feedback law of the form (2.15) - (2.16) was $\theta=1.7$; this behavior is shown by the dashed line. If in addition, one allows distributed control (at $\theta=1.7$ ) there is a loss in attenuation (but possibly better performance) as shown by the dotted line. However, by allowing control on the cable, the value of theta can be increased to $\theta=2.5$, greatly increasing disturbance attenuation and improving performance as shown by the solid line in Figure 2.2.

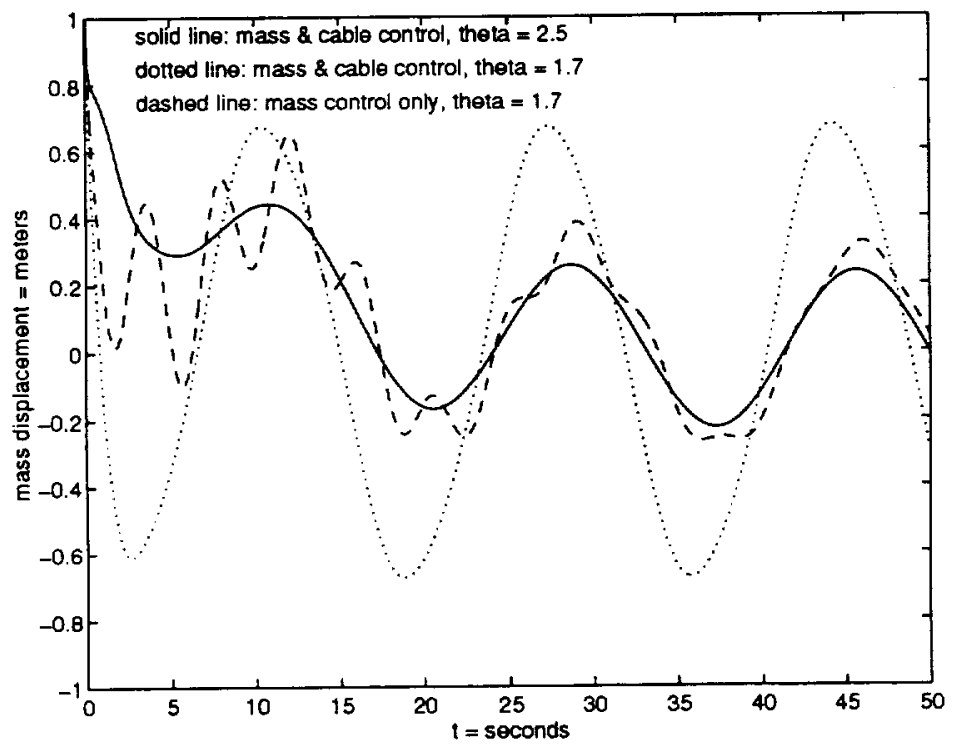

Figure 2.2: Displacement of the Mass under Three Types of Control.

This example clearly indicates the benefit of distributed control and MINMAX design. Moreover, as shown in [2] if one has explicit representation of the feedback operator $K_{\theta}$, then this can be used to design practical low order dynamic controllers (nonlinear compensators). In particular, it follows that since

$$
K_{\theta}=\left[\begin{array}{llll}
K_{\theta}^{11} & K_{\theta}^{12} & K_{\theta}^{13} & K_{\theta}^{14} \\
K_{\theta}^{21} & K_{\theta}^{22} & K_{\theta}^{23} & K_{\theta}^{24}
\end{array}\right]
$$

is bounded from $X$ to $H$, the control on the mass has the form

$$
\begin{aligned}
u_{2}(t)= & -\int_{0}^{\ell} \tau k_{s}(z) \frac{\partial}{\partial z} w(t, z) d z-\alpha_{1} k_{\alpha}(t, \ell) \\
& -\int_{0}^{\ell} \rho k_{v}(z) \frac{\partial}{\partial t} w(t, z) d z-m k_{m} \frac{\partial}{\partial t} w(t, \ell) .
\end{aligned}
$$


It is tempting to assume that one also has a representation of the form

$$
\begin{aligned}
u_{1}(t, \zeta) & =-\int_{0}^{\ell} \tau h_{s}(\zeta, z) \frac{\partial}{\partial z} w(t, z) d z-\alpha_{1} h_{\alpha}(\zeta)(t, \ell) \\
& -\int_{0}^{\ell} \rho h_{v}(\zeta, z) \frac{\partial}{\partial t} w(t, z) d z-m h_{m}(\zeta) \frac{\partial}{\partial t} w(t, \ell)
\end{aligned}
$$

In (2.18) $k_{s}(z)$ and $k_{v}(z)$ are functional gains corresponding to strain and velocity, respectively. Their existence and smoothness properties are assured by the Riesz Representation Theorem. For example, there is a $k_{s}(z) \in L_{2}(0, \ell)$ so that $K_{\theta}^{21}: H_{L}^{1} \rightarrow \mathbb{R}$ has the form

$$
\left[K_{\theta}^{21}\right] \phi(\cdot)=\int_{0}^{1} \tau k_{s}(z) \frac{d}{d z} \phi(z) d z
$$

However, on the surface all we know about $K_{\theta}^{13}: L_{2}(0,1) \rightarrow L_{2}(0,1)$ is that it is bounded. As noted above, the desire to find representations of such operators as integrals led to Grothendieck's work on nuclear spaces and the Schwartz Kernel Theorem. Recall that not all bounded linear operators on $L_{2}(0,1)$ (even if self-adjoint) have integral representations, as illustrated by the identity operator.

In [8] this issue was avoided by assuming a representation similar to (2.19) and then allowing generalized functions as kernels. This approach proved to be satisfactory for the one dimensional case considered therein, but does not apply to more general two and three dimensional hyperbolic problems. We present some results that lead to a representation of the form (2.19) when there is suitable damping in the system. Although this approach does not apply to the undamped case, the ideas can be extended to certain higher dimensional damped elastic systems. Moreover, we conjecture that the undamped problem in 3D systems will not yield a representation even of the type considered in [8].

We turn now to the simple $1 \mathrm{D}$ wave equation in order to state precise results and to keep this article at a reasonable length. Moreover, we restrict our presentation to LQR design $(\theta=0)$ since we can rely on existing literature to outline the results. Extensions to higher dimensional problems with $\theta>0$ will appear in a future paper.

\section{The Wave Equation}

Consider the wave equation with Kelvin-Voigt damping. Damping plays a key role in the design of controllers for hyperbolic systems. To illustrate this role, consider the LQR control problem for the wave equation defined by

$$
\begin{gathered}
\frac{\partial^{2}}{\partial t^{2}} w(t, s)=\frac{\partial^{2}}{\partial s^{2}} w(t, s)+\gamma \frac{\partial^{3}}{\partial s^{2} \partial t} w(t, s)+u(t, s), \\
0<s<1, \quad 0<t,
\end{gathered}
$$

with boundary conditions

$$
w(t, 0)=0, \quad w(t, 1)=0, \quad 0<t
$$

and cost function

$$
J(u)=\frac{1}{2} \int_{0}^{\infty} \int_{0}^{1}\left[\left|\frac{\partial}{\partial s} w(t, s)\right|^{2}+\left|\frac{\partial}{\partial t} w(t, s)\right|^{2}+|u(t, s)|^{2}\right] d s d t
$$

This problem is defined on the state space $X=H_{0}^{1}(0,1) \times L_{2}(0,1)$. As above, care must be used to define the system operators. In particular, let

$$
\mathcal{D}(A)=\left\{\left[\begin{array}{c}
w \\
v
\end{array}\right] \in X: w, v \in H_{0}^{1}(0,1), w+\gamma v \in H^{1}(0,1)\right\}
$$

and define $A$ by

$$
A\left[\begin{array}{c}
w \\
v
\end{array}\right]=\left[\begin{array}{c}
v(\cdot) \\
\frac{d}{d s}\left[\frac{d}{d s} w+\gamma \frac{d}{d s} v\right]
\end{array}\right]
$$


The control space is $U=L_{2}(0,1)$ and the control operator is defined from $U$ into $X=H_{0}^{1} \times L_{2}$ by

$$
B=\left[\begin{array}{c}
0 \\
I_{L_{2}}
\end{array}\right]
$$

Here, $\theta=0$ and $Q=R=I_{X}$. The LQR problem has a solution (even if $\gamma=0$ ) given by

$$
u(t, \cdot)=-K\left[\begin{array}{c}
w(t, \cdot) \\
\frac{\partial}{\partial t} w(t, \cdot)
\end{array}\right]
$$

where $K: H_{0}^{1}(0,1) \times L_{2}(0,1)$ has the form

$$
K=B^{*} P
$$

and $P$ satisfies the weak form of the Riccati equation (ARE) given by

$$
\langle P x, A z\rangle_{X}+\langle A x, P z\rangle_{X}-\left\langle B^{*} P x, B^{*} P z\right\rangle_{U}+\langle C x, C z\rangle_{Y}=0
$$

for all $x, y$ in $\mathcal{D}(A)$. For this second order system, $K$ takes the form

$$
K=\left[0, I_{L_{2}}\right] P,
$$

or equivalently,

$$
K=\left[P_{21}, P_{22}\right]
$$

where

$$
P=\left[\begin{array}{ll}
P_{11} & P_{12} \\
P_{21} & P_{22}
\end{array}\right]
$$

The operators $P_{21}: H_{0}^{1}(0,1) \rightarrow L_{2}(0,1)$ and $P_{22}: L_{2}(0,1) \rightarrow L_{2}(0,1)$ are bounded linear operators with $P_{22}^{*}=P_{22}$. The goal is to determine if there exist "nice" integral representations of these operators.

The following result is well known and may be found in [3].

Lemma 3.1 The operator $A$ generates an analytic semigroup $S(t)$ on $X$ and there exist $M>0, \omega>0$ with $\|S(t)\| \leq M e^{-\omega t}$.

Since $A$ generates a stable analytic semigoup, we can apply Theorem 2.1 (page 36) in [9] to obtain the following regularity result for $P$.

Lemma 3.2 There exists a self-adjoint, non-negative definite bounded linear transformation $P=P^{*}$ satisfying (3.29). Moreover, for each $\epsilon>0$, the operators $\left[A^{*}\right]^{1-\epsilon} P$ belong to $\mathcal{L}(X, X)$.

We note that Theorem 2.1 in [9] also states that $\epsilon$ can be set equal to zero if $A$ is self-adjoint, normal, or has a Riesz basis of eigenvectors. However, it is interesting to note that $A$ is neither normal nor self-adjoint. For this 1D problem, $A$ does have a Riesz basis. However, this property is not needed to establish the following representation.

Theorem 3.3 There exist functions $k_{21}(\cdot, \cdot), k_{22}(\cdot, \cdot)$ such that

(1) $k_{21}(\cdot, \cdot) \in L_{2}([0,1] \times[0,1]), k_{22}(\cdot, \cdot) \in L_{2}([0,1] \times[0,1])$,

(2) $k_{22}(\zeta, s)=k_{22}(s, \zeta)$,

(3) the mapping $t \rightarrow k_{21}(\xi, t)$ belongs to $H_{0}^{1}$ for almost all $\xi \in[0,1]$

and one has the representations

$$
\begin{aligned}
& {\left[P_{21} \phi\right](\zeta)=\int_{0}^{1} k_{21}(\zeta, t) \phi(t) d t, \quad \phi \in H_{0}^{1}} \\
& {\left[P_{22} \phi\right](\zeta)=\int_{0}^{1} k_{22}(\zeta, t) \phi(t) d t, \quad \phi \in L_{2}}
\end{aligned}
$$


The proof of Theorem 3.3 is rather tedious and will not be included here. However, we note that the proof is similar to the proof of Theorem 3.2 given in [7]. It relies on a classical theorem by Fullerton (see Theorem 6 in [5]). However, the specific structure of the $A$ operator combined with the special $B$ operator, $B=\left[\begin{array}{ll}0 & I_{X}\end{array}\right]^{T}$, is needed to carry out the proof for this hyperbolic case. Theorem 3.3 leads easily to the following representation.

Theorem 3.4 There exist functional gains $k_{v}(\cdot, \cdot)$ and $k_{s}(\cdot, \cdot)$

(1) $k_{s}(\cdot, \cdot) \in C([0,1] \times[0,1]), k_{v}(\cdot, \cdot) \in L_{2}([0,1] \times[0,1])$,

(2) $k_{v}(\zeta, z)=k_{v}(z, \zeta)$

(3) the mapping $z \rightarrow k_{s}(\zeta, z)$ belongs to $H^{2}$ for almost all $\zeta \in[0,1]$

and the LQR feedback control law has the representation

$$
u(t, \zeta)=-\int_{0}^{1} k_{s}(\zeta, z) \frac{\partial}{\partial z} w(t, z) d z-\int_{0}^{1} k_{v}(\zeta, z) \frac{\partial}{\partial t} w(t, z) d z
$$

Proof: Since $K: H_{0}^{1} \times L_{2} \rightarrow L_{2}$ is given by

$$
K\left[\begin{array}{l}
w(\cdot) \\
v(\cdot)
\end{array}\right]=-\int_{0}^{1} k_{21}(\zeta, z) w(z) d z-\int_{0}^{1} k_{22}(\zeta, z) v(z) d s
$$

let $k_{v}(\cdot, \cdot)=k_{22}(\cdot, \cdot)$. Integration by parts on the first integral yields (for $w(\cdot) \in H_{0}^{1}$ )

$$
\int_{0}^{1} k_{21}(\zeta, z) w(z) d z=-\int_{0}^{1}\left[\int_{0}^{z} k_{21}(\zeta, t) d t\right] w^{\prime}(z) d z
$$

The representation (3.35) follows, where

$$
k_{s}(\zeta, z)=-\int_{0}^{z} k_{21}(\zeta, t) d t
$$

and $z \rightarrow k_{s}(\zeta, z)$ belongs to $H^{2}$.

We turn now to some numerical experiments to illustrate the representation (3.35). In addition, we consider other damping models and present numerical results that clearly show the role that damping plays in the smoothness and existence of functional gains.

\section{Numerical Experiments}

We consider the wave equation with various forms of damping. We have the representation (3.35) for KelvinVoigt damping and similar results can be obtained for structural damping. However, as we shall see below, viscous damping is not sufficient to ensure the existence of $L_{2}$ functional gains even though the feedback operator exists and is bounded. We set $\gamma=.25$ and use standard piecewise linear finite elements to compute $k_{s}(\cdot, \cdot)$ and $k_{v}(\cdot, \cdot)$. In particular, we use finite elements to approximate the Riccati equation (3.29) and construct $K$ as defined by (3.30-3.32).

In order to show convergence of the scheme we compute $k_{s}^{N}(\cdot, \cdot)$ and $k_{v}^{N}(\cdot, \cdot)$ for Kelvin-Voigt damping. Here, $N$ represents the number of elements in the model (i.e., $[0,1]$ is partitioned into $(\mathrm{N}+1)$ subintervals). Figure 4.1 shows the convergence of $k_{s}^{N}(\cdot, \cdot)$ and $k_{v}^{N}(\cdot, \cdot)$ to $k_{s}(\cdot, \cdot)$ and $k_{v}(\cdot, \cdot)$, respectively. Observe that the convergence of $k_{s}^{N}(\cdot, \cdot)$ is very rapid.

Although we presented theoretical results for the case of Kelvin-Voigt damping only, Figure 4.2 shows that similar results hold for structural damping. In particular, an integral representation exists and the finite element approximations converge. It is important to note that the functional gain for strain, $k_{s}(\cdot, \cdot)$, remains smooth and is the same as the gain obtained with Kelvin-Voigt damping. However, there is a marked difference in the smoothness of $k_{v}(\cdot, \cdot)$. This functional gain has a sharper "peak" at $\zeta=z$ than the corresponding velocity gain for Kelvin-Voigt damping. Although we expect that for structural damping, $k_{v}(\zeta, \cdot)$ belongs to $H^{1}$, we conjecture that $t \rightarrow k_{v}(\zeta, t)$ is not $H^{2}$. 
Finally, for viscous damping, the numerical results shown in Figure 4.3 show that again, $k_{s}^{N}(\cdot, \cdot)$ is well behaved and yet $k_{v}^{N}(\cdot, \cdot)$ seems to be as singular measure concentrated at $\zeta=z$. Thus, we conjecture that the representation (3.35) does not hold for the wave equation with viscous damping for any $L_{2}$ function $k_{v}(,$,$) . Similar results were noted in [8] for undamped beam equations and in [7] for parabolic equations$ with highly unbounded input operators.

The numerical results also indicate that the strain functional gains are independent of the damping model. As shown in Figure $4.4, k_{s}^{N}(\cdot, \cdot)$ does not change as the damping model changes. Thus, it seems from the numerical experiments that damping has the most impact on the existence and smoothness of $k_{v}(\cdot, \cdot)$.

\section{Conclusions}

In this paper we have provided an integral representation theorem for the LQR feedback operator for the 1D wave equation with Kelvin-Voigt damping and fully distributed control. The motivation for this effort comes from the problem of designing suboptimal low order dynamic compensators and for optimal sensor location. Although the theoretical results presented here are restricted to problems with Kelvin-Voigt damping, the numerical results suggest that more general results are available. Also, these same numerical results indicate that once the damping is insufficient to ensure the analyticity of the control system, the existence of $L_{2}$ functional gains is in doubt. In particular, the solution of the Riccati equation may not be Hilbert-Schmidt.

The LQR problem for distributed parameter systems has received considerable attention during the past ten years. However, problems in which $B, Q$ and $R$ are all non-compact have not been fully explored. Temam [12] considers the differential Riccati equation with $B=R=I$, but assumes that $Q$ is Hilbert-Schmidt. Similar results are found in $[4,6]$. Finally, as the numerical evidence suggests, many theoretical issues are not yet settled.

\section{References}

[1] J. A. BURNS and B. B. KING, "A Note on the Regularity of Solutions of Infinite Dimensional Riccati Equations," Appl. Math. Lett., v. 7, 1994, pp. 13-17.

[2] J. A. BURNS and B. B. KING, "Optimal Sensor Location for Robust Control of Distributed Parameter Systems," Proc. of the 33rd IEEE Control and Decision Conference, Dec. 1994, pp. 3967-3972.

[3] G. CHEN and J. ZHOU, Vibration and Damping in Distributed Systems, Vol I. \& II, Studies in Advanced Mathematics, CRC Press, Boca Raton, 1993.

[4] A. DE SANTIS, A. GERMANI and L. JETTO, "Approximation of the Algebraic Riccati Equation in the Hilbert Space of Hilbert-Schmidt Operators," SIAM J. Control Optim., v. 31, 1993, pp. 847-874.

[5] R. E. FULLERTON, "Linear Operators with Range in a Space of Differentiable Functions," Duke Math. Journal, v. 13, 1946, pp. 269-280.

[6] A. GERMANI, L. JETTO and M. PICCIONI, "Galerkin Approximation for Optimal Filtering of Infinite Dimensional Linear Systems," SIAM J. Control Optim., v. 26, 1988, pp. 1287-1305.

[7] B. B. KING, "Existence of Functional Gains for Parabolic Control Systems" Proc. Computation and Control IV, 1995.

[8] V.D. LUPI, H.M. CHUN, J.D. TURNER, "Distributed Control without Mode Shapes or Frequencies," Adv. in the Astro. Sci., v. 76, 1991, pp. 447-470.

[9] 1. LASIECKA and R. TRIGGIANI, Differential and Algebraic Riccati Equations with Application to Boundary/Point Control Problems: Continuous Theory and Approximation Theory, Lecture Notes in Control and Information Sciences, v. 164, Springer-Verlag, Berlin, Heidelberg, 1991. 
[10] C. MCMILLAN and R. TRIGGIANI, "Min-Max Game Theory and Algebraic Riccati Equations for Boundary Control Problems with Continuous Input-Solution Map, Part II: General Case, Applied Math. and Optimization, v. 29, 1994, pp. 1-65.

[11] A. H. NAYFEH, J. F. NAYFEH, and D. T. MOOK, "On Methods for Continuous Systems with Quadratic and Cubic Nonlinearities", Nonlinear Dynamics, v. 3, 1992, pp. 145-162.

[12] R. TEMAM, "Sur l'equation de Riccati associée à des opérateurs non bornés, en dimension infinie", $J$. Func. Anal., v. 7, 1971, pp. 85-115.

$$
\text { k_s(...), N = } 8
$$

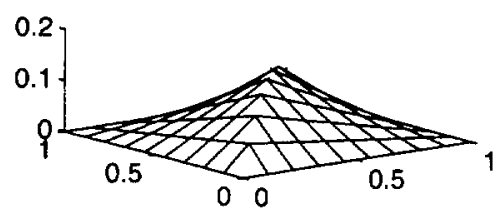

$$
\text { k_s(.., }, N=16
$$

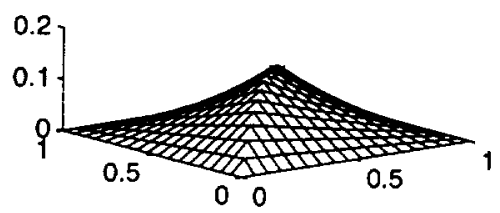

$$
\text { k_s(...), N }=32
$$

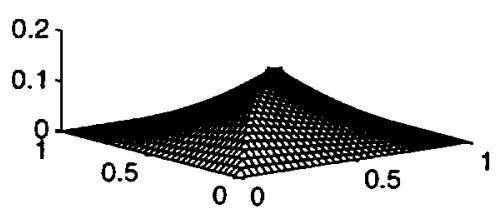

k_v(...), N = 8

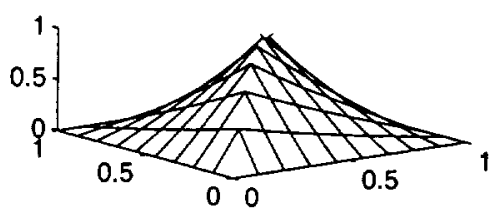

k_v(.,.), N = 16

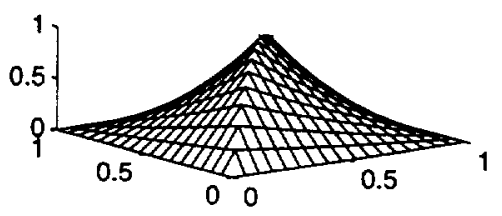

$k \_v(. .),. N=32$

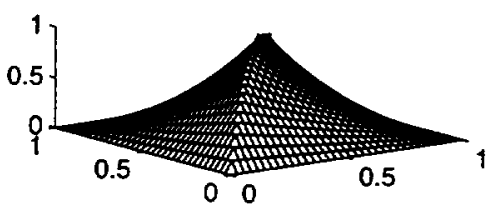

Figure 5.1: Convergence of the Functional Gains, Kelvin-Voigt Damping. 


$$
k \_s(\ldots), N=8
$$

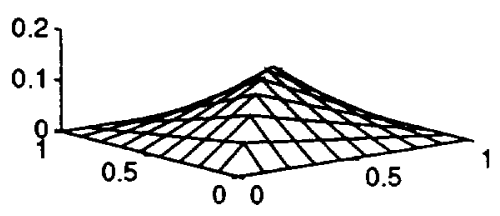

$$
k \_s(. .), N=16
$$

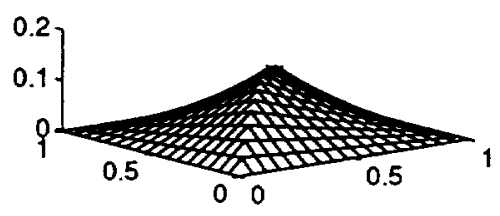

$$
\text { k_s(...), N = } 32
$$

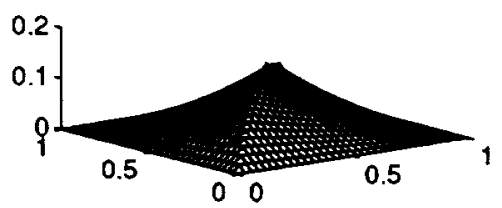

$k \_v(.,),. N=8$

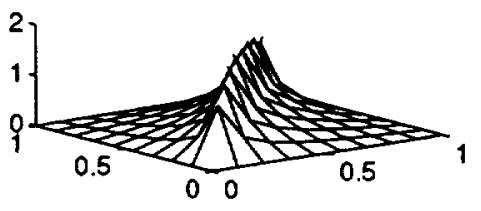

$k \_v(.),, N=16$

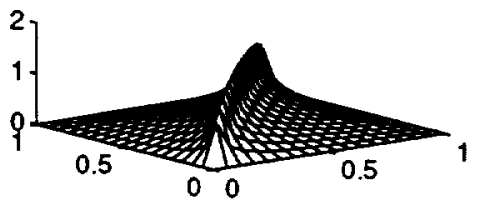

$k \_v(.),. N=32$

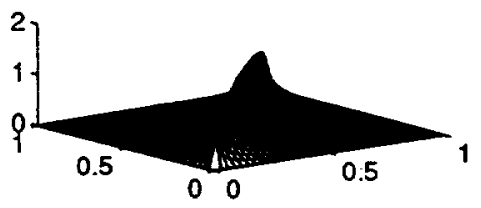

Figure 5.2: Convergence of the Functional Gains, Structural Damping.

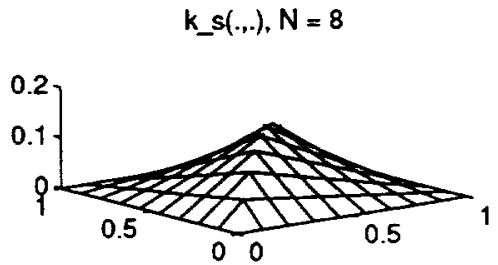

$k \_s(.,),. N=16$

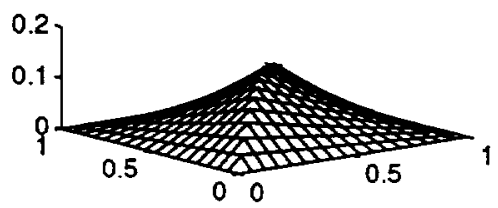

$k \_s(.),, N=32$

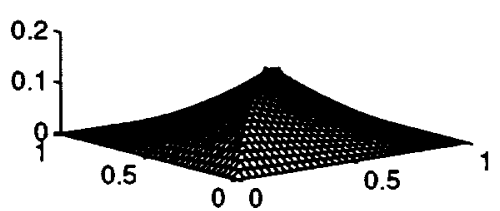

$k \_v(.,),. N=8$

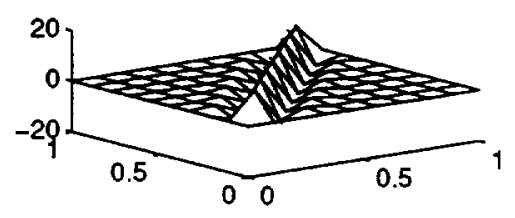

k_v(.,.), $N=16$

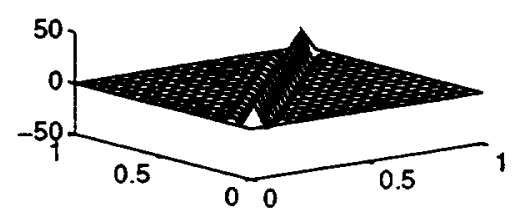

$k \_v(.),. N=32$

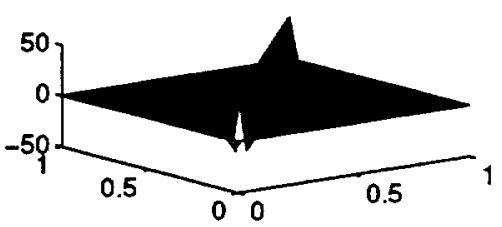

Figure 5.3: Convergence of the Functional Gains, Viscous Damping. 

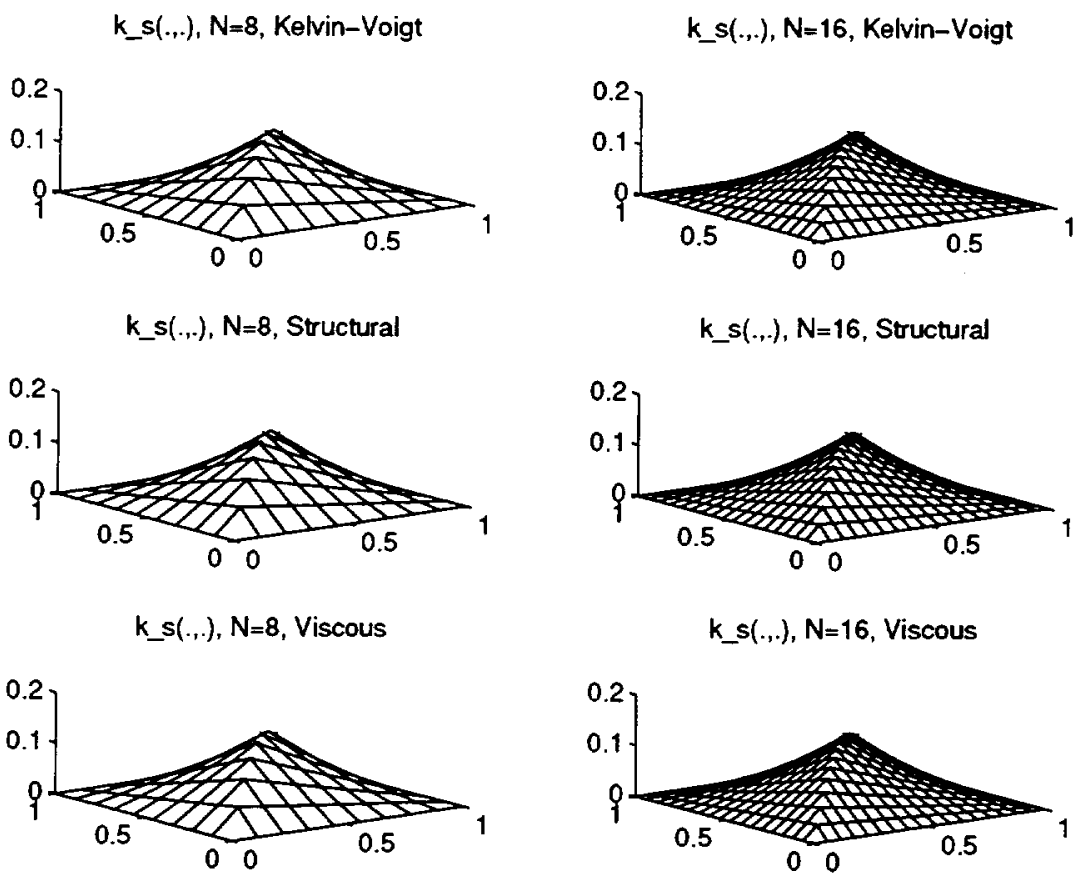

Figure 5.4: Structural Gains for Various Damping Types, $\mathrm{N}=8,16$. 



\section{REPORT DOCUMENTATION PAGE}

Public reporting burden for this collection of information is estimated to average 1 hour per response, including the time for reviewing instruetions, searching existing data sources, Public gathering and maintaining the data needed, and completing and reviewing Washington Headouarters Services, Directorate for Information Operations and Reports, 1215 Jefferson (1) Office of Management and Budget. Paperwork Reduction Project (0704-0188). Washington, DC 20503.

\begin{tabular}{|l|l|l}
\hline 1. AGENCY USE ONLY(Leave blank) & $\begin{array}{l}\text { 2. REPORT DATE } \\
\text { May } 1995\end{array}$ & $\begin{array}{l}\text { 3. REPORT TYPE AND DATES COVERED } \\
\text { Contractor Report }\end{array}$ \\
\hline
\end{tabular}

4. TITLE AND SUBTITLE

REPRESENTATION OF FEEDBACK OPERATORS

FOR HYPERBOLIC SYSTEMS

C NAS1-19480

WU 505-90-52-01

6. AUTHOR(S)

John A. Burns

Belinda B. King

7. PERFORMING ORGANIZATION NAME(S) AND ADDRESS(ES)

Institute for Computer Applications in Science

and Engineering

Mail Stop 132C, NASA Langley Research Center

Hampton, VA 23681-0001

9. SPONSORING/MONITORING AGENCY NAME(S) AND ADDRESS(ES)

National Aeronautics and Space Administration

Langley Research Center

Hampton, VA 23681-0001

8. PERFORMING ORGANIZATION

REPORT NUMBER

ICASE Report No. 95-45

10. SPONSORING/MONITORING AGENCY REPORT NUMBER

NASA CR-198171

ICASE Report No. 95-45

11. SUPPLEMENTARY NOTES

Langley Technical Monitor: Dennis M. Bushnell

Final Report

To appear in the Conference on Computation and Control

12a. DISTRIBUTION/AVAILABILITY STATEMENT

12b. DISTRIBUTION CODE

Unclassified-Unlimited

Subject Category 64

13. ABSTRACT (Maximum 200 words)

We consider the problem of obtaining integral representation of feedback operators for damped hyperbolic control systems. We show that for the wave equation with Kelvin-Voigt damping and non-compact input operator, the feedback gain operator is Hilbert-Schmidt. This result is then used to provide an explicit integral representation for the feedback operator in terms of functional gains. Numerical results are given to illustrate the role that damping plays in the smoothness of these gains.

14. SUBJECT TERMS

Feed back Control; Hyperbolic Systems
15. NUMBER OF PAGES

13

16. PRICE COOE $\mathrm{A} 03$

18. SECURITY CLASSIFICATION 19. SECURITY CLASSIFICATION OF ABSTRACT

Unclassified

\section{OF REPORT}

Unclassified

NSN 7540-01-280-5500

\begin{tabular}{|c|c|} 
& $\begin{array}{c}\text { 15. NUMBER OF PAGES } \\
13\end{array}$ \\
\cline { 2 - 2 } & $\begin{array}{c}\text { 16. PRICE CODE } \\
\text { A03 }\end{array}$ \\
\hline $\begin{array}{l}\text { 19. SECURITY CLASSIFICATION } \\
\text { OF ABSTRACT }\end{array}$ & $\begin{array}{l}\text { 20. LIMITATION } \\
\text { OF ABSTRACT }\end{array}$ \\
\hline $\begin{array}{l}\text { Standaad Form 298(Rev. 2-89) } \\
\text { Prescribed by ANSI Std. Z39-18 } \\
\text { 298-102 }\end{array}$
\end{tabular}


